\title{
RADIO ASTRONOMY TECHNIQUES OF OBSERVING MAGNETIC FIELDS: THE GALAXY
}

\author{
RICHARD WIELEBINSKI \\ Max-Planck-Institut für Radioastronomie, Auf dem Hügel 69, W-5300 Bonn 1, FRG
}

October 29, 1992

\section{Introduction}

Optical techniques were first used to detect magnetic fields in cosmic objects. Hale used the Zeeman effect to detect solar magnetic fields in 1908. Babcock extended the Zeeman technique to show the existence of magnetic fields in Ap stars in 1946. Optical polarization observations were made as early as 1920 by W.F. Meyer who observed the Hubble variable nebula NGC 2261. Polarization observations of the Andromeda nebula were made by Öhman in 1942 . However at first the interpretation of these optical polarization observations was in terms of scattered light only. The theoretical work of Davis and Greenstein suggested that optical polarization could also be due to dust grains aligned in magnetic fields. Observations of Hiltner and Hall supported this interpretation. Extensive surveys of starlight polarization were made by many observers giving information about nearby magnetic fields in the Galaxy. Optical polarization observations of galaxies gave some information on the Magellanic Clouds and other nearby objects but due to lack of sensitivity progress was slow.

Radio astronomy offers a greater number of techniques to study magnetic fields in various cosmic objects. The Zeeman effect is observable in several molecular lines at radio wavelengths. Hence information about magnetic fields in various species of molecular clouds in the Galaxy can be obtained. The radio synchrotron radiation is emitted with a high degree of polarization and hence can be used as a probe of magnetic fields. Multi-frequency maps of polarized emission can give the orientation of the magnetic fields in such objects as supernova remnants, nearby galaxies and radio galaxies. The Faraday rotation due to either Galactic or extragalctic magnetic fields can be measured allowing the determination of the fields in the line of sight. Pulsars, which are highly linearly polarized, can be used as a unique probe of Galactic magnetic fields.

In this review some of the details of the techniques used in radio astronomy will be described with particular reference to the results on the magnetic fields in the Galaxy.

\section{The Zeeman Effect}

The Zeeman effect is a direct method of measuring magnetic fields. It depends on the fact that a magnetic field causes a split of a line due to the conservation of angular momentum on the field direction in an atom or a molecule. The unshifted component corresponds to a transition that leaves the projection of the angular 
Fig. 1. The Zeeman effect. A line is split by a parallel magnetic field into two opposite circular components. The difference gives the typical 'S' signature.

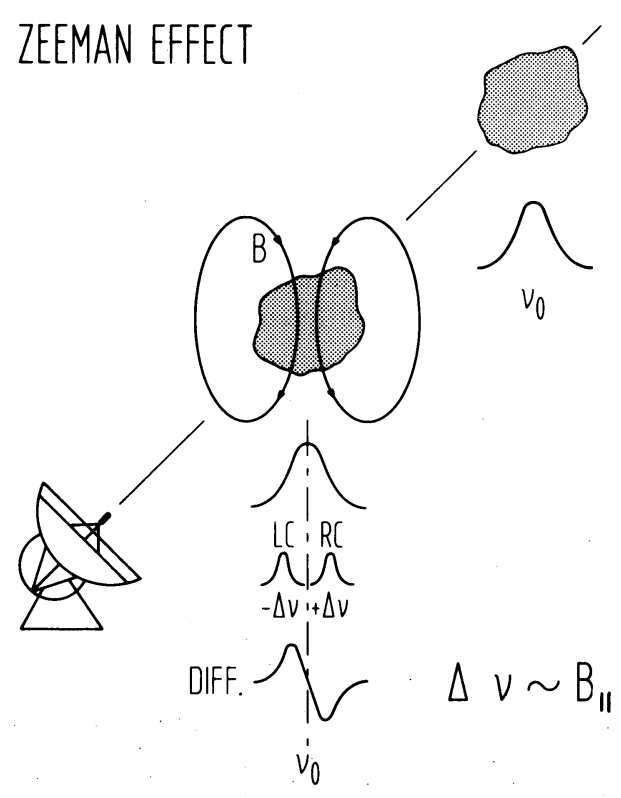

momentum unchanged. The shifted components are right and left-circularly polarized (see Figure 1). The magnitude of the frequency shift depends on the magnetic field strength and the wavelength of the line. In the optical range the line width can mask any shifts and the limit of measurable fields is $B>10^{3}$ Gauss. In the radio range we use such lines as $\mathrm{HI}$ or $\mathrm{OH}$ where the shifts are $2.8 \mathrm{~Hz} / \mu \mathrm{Gauss}$ and $3.8 \mathrm{~Hz} / \mu$ Gauss respectively. Field strengths of $B \sim 10 \mu \mathrm{Gauss}$ have been measured. The Zeeman effect has also been measured in $\mathrm{H}_{2} \mathrm{O}$ masers (i.e. in regions of dense gas) in our Galaxy where field strengths $B>10^{3}$ Gauss were found.

\section{Synchrotron Emission as a Signature of Magnetic Fields}

The nonthermal (synchrotron) emission, which is predominant in the $\mathrm{m}$ - to $\mathrm{cm}$ wavelength range, is generated in magnetic fields. A relativistic electon on entering a magnetic field is forced into a helical path (see Figure 2) generating linearly polarized radiation. This radiation can be up to $75 \%$ linearly polarized in a homogenous emitting region allowing the determination of the orientation of the field perpendicular to the line of sight. Observations must be made at sufficiently high radio frequencies to ensure that the intervening medium does not change the polarization angle.

The change of the polarization angle in the intervening medium is due to the Faraday effect (see Figure 3). A linearly polarized wave traversing a magnetoionic medium is split into two circularly polarized components. The propagation speed of the two components is different so that on emergence of the wave from the medium a change of the polarization angle is observed. The amount of change is proportional to the product of thermal electron content, path length and the parallel component of the magnetic field. 


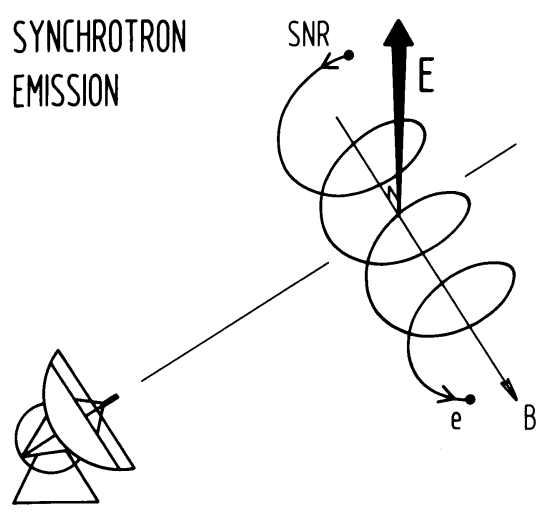

Fig. 2. The synchrotron emission can be detected through direct polarization measurements. The emitted 'E'-vector is perpendicular to the magnetic field direction.

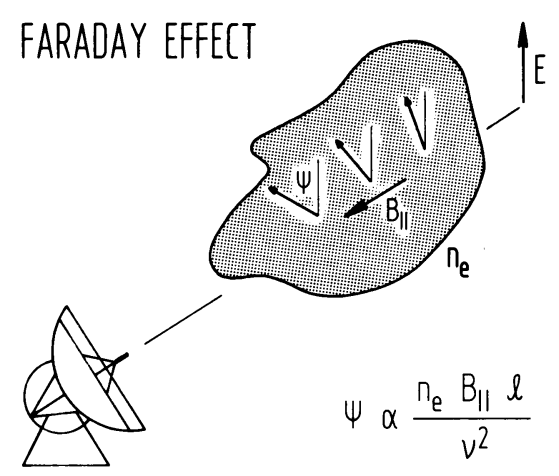

Fig. 3. The Faraday effect leads to the rotation of the emitted ' $E$ '-vector by the magnetic field in the line of sight.

The synchrotron intensity is also a signature of magnetic field. The earliest radio astronomy observations made by Jansky and Reber were in fact of the nonthermal synchrotron radiation. By applying an equipartition argument (between magnetic field and cosmic rays) an estimate of the field strength is possible (e.g. see Moffat 1975; Pacholczyk 1976).

\section{The Discovery of Magnetic Fields in the Galaxy}

The observations of Hiltner (1949) and Hall (1949) used in conjunction with the interpretation of Davis and Greenstein (1951) gave strong support to the suggestion of the existence of magnetic fields in the Galaxy. Collection of data on starlight polarization continued, with substantial catalogues being published by Hiltner (1956), Behr (1959), and others, culminating in the collected catalogue of 1800 stars by Mathewson and Ford (1970). The interpretation was not easy since most of the observed stars were relatively nearby. Some areas of substantial alignment of the vectors could however be identified. One of the interpretations put forward by Mathewson and Nicholls (1968) was that the local field was helical in accordance with the theoretical predictions of Hoyle and Ireland (1961). Photopolarimetry was also used in the searches for magnetic fields, in particular in galaxies.

In the meantime observations of discrete objects were more successful in proving the presence of magnetic fields in the Galaxy. The search for polarization was initiated by the suggestion of Shklovsky (1953) that the radiation in the Crab nebula was due to the synchrotron process in all spectral ranges. The optical observations of the Crab nebula by Dombrovsky (1954) showed that the starlight was indeed highly polarized. Follow-up observations by Oort and Walraven (1956) and later by Woltjer (1957) showed an incredibly well organised polarization which could only be attributed to magnetic fields. Radio polarization was detected in the Crab 
nebula by Mayer et al. (1957).

Radio polarization of the Galactic emission was first reported by Westerhout et al. (1962) and Wielebinski et al. (1962), both observations being made at 408 $\mathrm{MHz}$. The direction of highly aligned field was found to be $l=140^{\circ}, b=10^{\circ}$. In this direction there was a good agreement with the optical results indicating that the magnetic field was aligned parallel to the spiral arm. The surveys that followed did not give results that were easy to interpret.

The radio polarization of discrete extragalactic sources allows us to study Galactic magnetic fields through their Faraday rotation within the Galaxy. The first reports of the polarization of radio galaxies came from Mayer et al. (1962) Gardner and Whiteoak (1962) and Bracewell et al. (1962). The studies of the Rotation Measure (RM) of a large number of sources was applied to study the Galactic magnetic fields (e.g. Vallée and Kronberg 1973).

Pulsars are highly polarized and very suitable to measure the magnetic field between the source and the observer. In addition to the RM the Dispersion Measure can be determined from the timing of pulse arrival at various frequencies. This allows a unique determination of the magnetic field (e.g. Manchester 1974).

The Zeeman effect, the most direct method of measurement, was at first very elusive at radio frequencies. After numerous attempts Verschuur (1968) detected the Zeeman effect in absorption in the direction of Galactic HI clouds. The detection of the Zeeman effect in the $\mathrm{OH}$ molecule was first reported by Crutcher and Kazes (1983). Strong magnetic fields in interstellar $\mathrm{H}_{2} \mathrm{O}$ maser clumps were detected by Fiebig and Güsten (1989).

\section{The Present 'State' of the Magnetic Fields in the Galaxy}

The low-frequency all-sky radio continuum surveys can be used to delineate the total magnetic field. Such work by Phillips et al. (1981) and Beuermann et al. (1985) suggested that the magnetic fields are closly correlated with the spiral arm pattern. The field strengths that are deduced are some ugauss in both the random and turbulent component, up to $10^{-5}$ Gauss total.

Polarization mapping of Galactic loops (Spoelstra 1971) and of Galactic regions (Spoelstra 1984) showed that even the lower radio frequencies (up to $1400 \mathrm{MHz}$ ) can be used to study the nearby magnetic fields. Observations at $2.7 \mathrm{GHz}$ by Junkes et al. (1987) showed that polarization can be used to distinguish supernova remnants in the Galactic plane (see Figure 4). In this $2.7 \mathrm{GHz}$ survey it also became clear that this technique allows the detection of polarized emission from the inner parts of the Galaxy.

The Galactic centre turns out to be a most unusual object with spectacular magnetic fields. Observations at $333.1 \mathrm{MHz}$ by Anantharamaiah et al. (1991) with the VLA showed thin filaments perpendicular to the palne. Direct polarization observations at $10.6 \mathrm{GHz}$ by Seiradakis et al. (1989) and at $8.4 \mathrm{GHz}$ by Haynes et al. (1992) showed directly vertical magnetic fields. Since very high RMs are seen in the Galactic centre magnetic field strengths of $B>100 \mu$ Gauss have been suggested. In fact some claims of fields as high as tens of milligauss have been made.

The studies of RMs of extragalactic sources proceeded from the early beginnings 


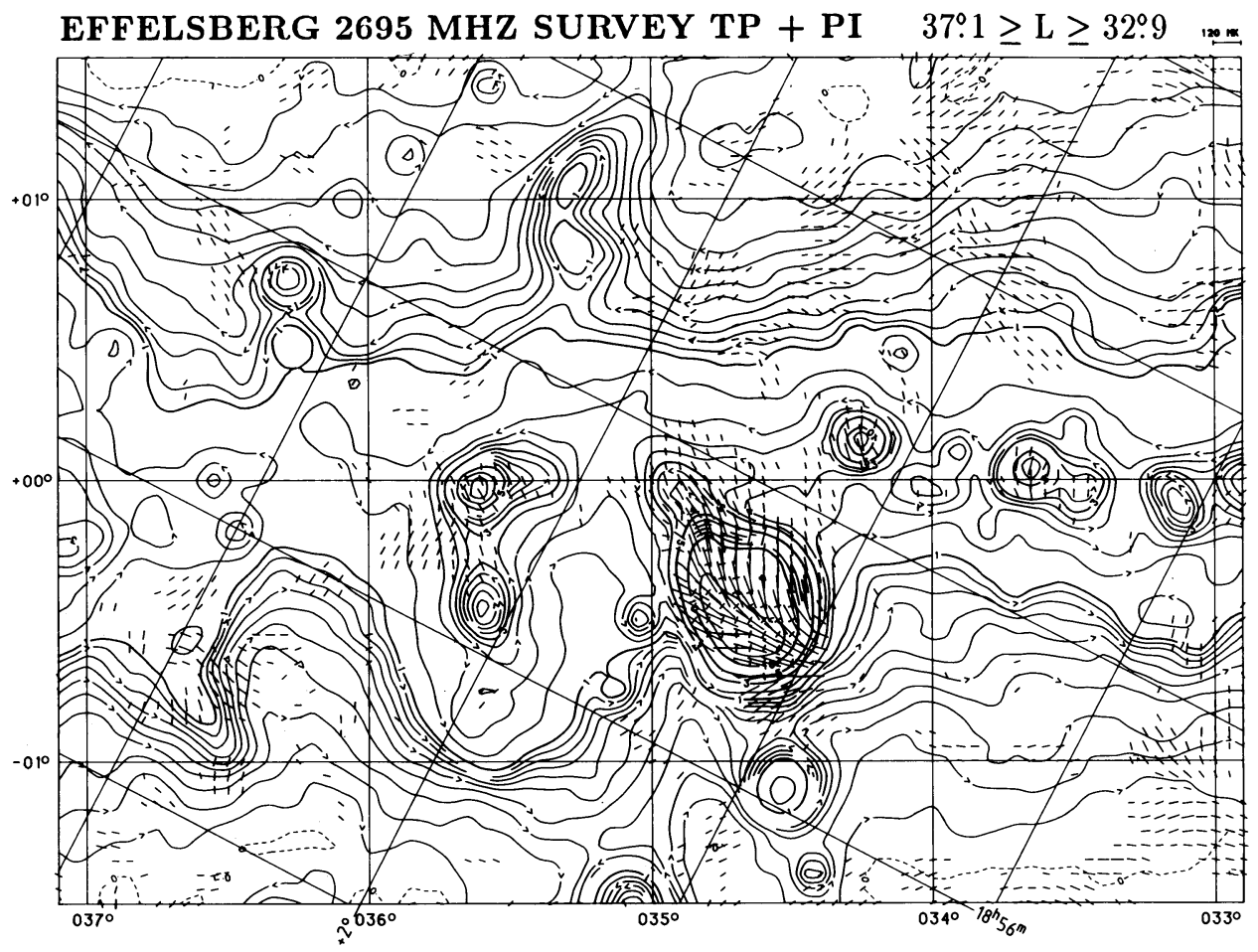

Fig. 4. A section of the $11 \mathrm{~cm}$ Galactic plane polarization survey of Junkes et al. (1987).

to a collection of some 1000 sources now. Simard-Normandin et al. (1981) who studied 555 sources suggested an aligned local field and a number of 'local features'. The most recent collection of RMs (P.P. Kronberg private communication; Figure 5 ) shows a band of high RM sources in the Galactic plane. However some areas of high RM are seen at high galactic latitudes indicating the presence of localised thermal material as well as of magnetic fields. The early quasi-symmetry of positive and negative RMs which suggested an aligned local field has dissapeared. The RM data gave a hint of a reversal of the magnetic field direction in the inner Galaxy. This fact was used before by Sofue and Fujimoto (1983) to suggest hat the Galaxy has a bisymmetrical magnetic field. Several studies of Vallee (1991 and references therein) dealt with the structure of the Galactic magnetic field from rotation measure studies. There are several studies of selected areas (e.g. Bignell et al. 1988; McLeod et al. 1988) which suggest that there are sudden field reversals which are seen on the scale of a few degrees.

Pulsars are very important in their ability to give unique information about the magnetic field in the line of sight. Studies by Lyne and Smith (1989) of 185 pulsars suggested that the local field is directed towards $l=90^{\circ}$ and $2-3 \mu$ Gauss in strength. The distribution of RMs is similar to that derived from extragalactic sources, suggesting that we observe pulsars in the whole Galaxy. Rand and Kulkarni 


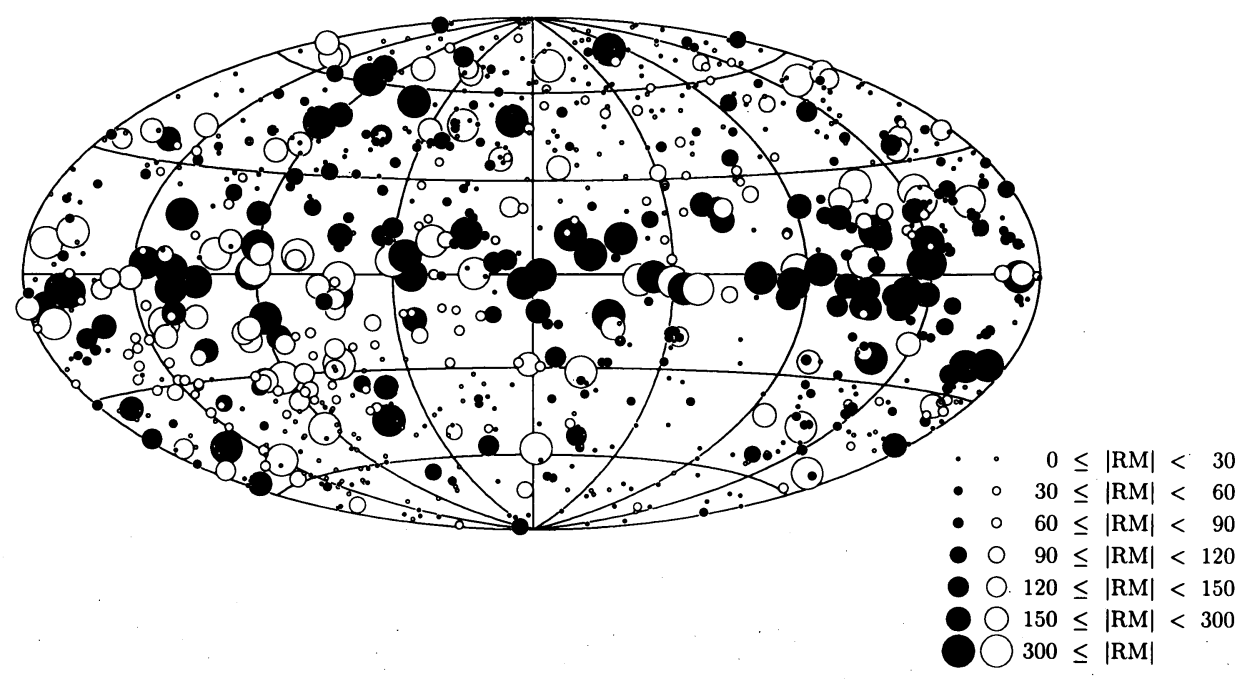

Fig. 5. Collected rotation measures of 976 extragalactic radio sources (P.P. Kronberg, private communication).

(1989) have made studies of pulsars in selected areas suggesting a somewhat lower value of the local magnetic field strength. A stronger magnetic field in the direction of the North Polar spur was deduced. A field reversal towards the inner Galaxy was confirmed. A more recent study of pulsar distances, interstellar scattering and the distribution of dispersion measures with respect to distance by Taylor and Cordes (1992) abandon the axisymmetric Galaxy assumption and show that the spiral arms determine the magnetic field pattern.

The Zeeman effect studies have given detections in many more sources. The early pioneering work of Verschuur was followed by large surveys (e.g. Heiles 1988; 1989). The number of measurements now exceeds 300 , although most remain unpublished (Heiles 1990). Also Verschuur (1989) has continued to observe the Zeeman effect in HI clouds giving check possibilities for the observations of Heiles. In the $\mathrm{OH}$ line further observations by Crutcher, Troland and Kazes (see Crutcher 1988) gave us many new results. Not only single dishes but also aperture synthesis telescopes have now been used to study the magnetic fields both in $\mathrm{HI}$ and $\mathrm{OH}$ clouds. The work of Fiebig and Güsten on $\mathrm{H}_{2} \mathrm{O}$ masers and other molecular lines continues.

\section{Conclusion}

Our knowledge about the magnetic fields in our Galaxy is considerable, but not precise. We see many details, but cannot delineate much of the information because of the 'insider' view of the Galaxy. Observations of other galaxies will obviously help us in understanding our own situation. Then we can return to the steadily 
accumulating data on our Galaxy and try to say what sort of magnetic field we live in.

\section{References}

Anantharamiah, K.R., Pedlar, A., Ekers, R.D. and Goss, W.M.: 1991, MNRAS 249, 262

Behr, A.: 1959, Nachr. Akad. Göttingen 7

Beuermann, K., Kanbach, G. and Berkhuijsen, E.: 1985, $A \& A$ 153, 17

Bignell, R.C., Simard-Normandin, M. and Vallée, J.P.: 1988, ApJS 67, 279

Bracewell, R.N., Cooper, B.F.C. and Cousins, T.E.: 1962, Nature 165, 1286

Crutcher, R.M.: 1988, in R.L. Dickman et al., ed(s)., Molecular Clouds in the Milky Way and External Galaxies, Springer: Berlin, p. 105

Crutcher, R.M. and Kazes, I.: 1983, $A \& A$ 125, L23

Dombrovsky, V.A.: 1954, Dokl. Akad. Nauk UdSSR 94, 1021

Fiebig, D. and Güsten, R.: 1989, $A \cup A$ 214, 333

Gardner, F.F. and Whiteoak, J.B.: 1962, Phys. Rev. Lett. 9, 197

Hall, J.S.: 1949, Science 109, 166

Haynes, R.F. et al.: 1992, $A \mathcal{B} A$ 264, 500

Heiles, C.: 1988, $A p J$ 334, 321

Heiles, C.: 1989, $A p J$ 336, 808

Heiles, C.: 1990, in R. Beck et al., ed(s)., Proc. IAU Symp. 140, Galactic and Intergalactic Magnetic Fields, Kluwer: Dordrecht, p. 35

Hiltner, W.A.: 1949, Science 109, 471

Hiltner, W.A.: 1958, $A p J$ 128, 9

Hoyle, F. and Ireland, J.G.: 1961, MNRAS 122, 462

Junkes, N., Fürst, E. and Reich, W.: 1987, $A \& A S 69,451$

Lyne, A.G. and Smith, F.G.: 1989, MNRAS 237, 533

Manchester, R.N.: 1974, $A p J$ 188, 637

Mathewson, D.S. and Nicholls, D.C.: 1968, $A p J$ 154, L11

Mathewson, D.S. and Ford, V.L.: 1970, Mem. R. Astron. Soc. 74, 139

Mayer, C.H., McCullough, T.P. and Sloanaker, R.M.: 1957, $A p J$ 126, 468

Mayer, C.H., McCullough, T.P. and Sloanaker, R.M.: 1962, ApJ 135, 656

McLeod, J.M., Vallée, J.P. and Broten, N.W.: 1988, $A \mathcal{B} A S$ 74, 63

Moffat, A.T.: 1975, in A. Sandage et al., ed(s)., Stars and Stellar Systems Vol. 9, Galaxies and the Universe, Chicago Univ. Press: Chicago, p. 211

Pacholczyk, A.G.: 1970, Radio Astrophysics, Freeman: San Francisco

Phillips, S. et al.: 1981, $A \& A 103,405$

Rand, R.J. and Kulkarni, S.R.: 1989, $A p J$ 343, 760

Seiradakis, J.H. et al.: 1989, $A \mathscr{E} A S$ 81, 291

Shklovsky, I.S.: 1953, Dokl. Akad. Nauk UdSSR 90, 1983

Simard-Normandin M., Kronberg, P.P. and Button, S.: 1981, ApJS 45, 97

Sofue, Y. and Fujimoto, M.: 1983, $A p J$ 265, 722

Spoelstra, T.A.Th.: 1971, $A \& A$ 13, 237

Spoelstra, T.A.Th.: 1984, $A \mathcal{E} A$ 135, 238

Taylor, J.H. and Cordes, J.M.: 1992, NAIC Preprint No. 297

Vallée, J.P.: 1991, ApJ 366, 450

Vallée, J.P. and Kronberg, P.P.: 1973, Nature 246, 49

Verschuur, G.L.: 1968, Phys. Rev. Lett. 21, 775

Verschuur, G.L.: 1989, $A p J$ 339, 163

Westerhout, G., Seeger, Ch.L., Brouw, W.N. and Tinbergen, J.: 1962, B.A.N. 16, 187

Wielebinski, R., Shakeshaft, J.S. and Pauliny-Toth, I.I.K.: 1962, Observatory 82, 187

Woltjer, L.: 1957, B.A.N. 13, 301 